\title{
Uso de los espacios virtuales para la docencia
}

\author{
Using virtual environments for teaching
}

\section{Sr. Editor:}

Hemos leído atentamente el artículo titulado "Uso de los espacios virtuales para la docencia en cursos de pregrado en Medicina" (1) y comprendemos la gran importancia actual de estudios como éste para el progreso de la educación.

Somos conscientes que para evaluar el impacto del uso de los espacios virtuales para la docencia (EVD) en el rendimiento académico, es necesario realizar estudios más complejos, como el realizado por PeñaSarmiento M. y col (2) en el que se considera "la evaluación del aprendizaje en los estudiantes y de la percepción del trabajo adelantado con docentes y estudiantes". Sin embargo, hay algunos aspectos importantes que no han sido considerados en el estudio de Orellano C (1). A continuación mencionaremos algunos, basados en nuestra experiencia personal como estudiantes universitarios.

Con respecto a los objetivos mínimos a alcanzar, si bien es cierto, el grado de cumplimiento de la "publicación de las presentaciones de las clases presenciales" se logró, este no da información sobre la puntualidad de la publicación de las clases en el EVD, orden secuencial, ni el control de calidad de las mismas. Por ejemplo, en algunos cursos la publicación de las clases en el EVD pudo haber sido realizado varios días después de su dictado, o las clases colocadas no eran las mismas que las dictadas en el aula o estaban incompletas (control de calidad).

Algo similar pudo ocurrir en la carpeta "otros archivos", en el que se colocan artículos científicos que deben revisar los estudiantes. Además, siempre existe la posibilidad que algún archivo puesto en el EVD tenga problemas para abrir o descargarlo. Por esta razón, es necesario que haya control de la calidad del material disponible en el EVD, para garantizar la accesibilidad y la calidad del contenido.

\author{
Luis Enrique Frisancho-Morales \\ Estudiante de Medicina. Facultad de Medicina \\ Alberto Hurtado, Universidad Peruana Cayetano \\ Heredia, Lima, Perú. \\ Albert Fernando Gonzales-Salcedo \\ Estudiante de Medicina. Facultad de Medicina \\ Alberto Hurtado, Universidad Peruana Cayetano \\ Heredia, Lima, Perú.
}

\section{REFERENCIAS BIBLIOGRÁFICAS}

1. Orellano C. Uso de los espacios virtuales para la docencia en cursos de pregrado de Medicina. Rev Med Hered. 2012; 23(3):188-192.

2. Peña-Sarmiento M., Avendaño-Prieto BL. Evaluación de la implementación del aula virtual en una Institución de Educación Superior. Suma Psicológica. 2006; 13(2): 173-192.

\section{Respuesta del Autor}

\section{Author / Editor Response}

De acuerdo a los comentarios realizados sobre el artículo (1), puedo señalar lo siguiente:

El objetivo del estudio no fue evaluar indicadores de calidad respecto a los usos de los diferentes tipos de recursos y actividades disponibles en los EVD y tampoco obtener información sobre las percepciones de los usuarios, aspectos mencionados por los redactores de la carta.

En el estudio de Peña-Sarmiento M (2), no se indica el software utilizado y si existieron algunas condiciones generales que deberían ofrecer las aulas virtuales de las asignaturas. Esto es importante para el análisis de resultados ya que podrían aparecer algunos 
sesgos que dependen de las habilidades y experiencia de cada docente en cuanto al uso de las tecnologías de información y comunicaciones para la educación.

Sobre los problemas de descarga de archivos, mientras el docente haya realizado los pasos correctos para generar los enlaces a los mismos, no dependen del aula virtual sino de las características de conexión o la configuración del explorador. En el caso de errores para abrirlos, podría suceder que la computadora del alumno no cuente con el software adecuado, siendo esta situación ajena a los EVD.

Como se menciona en el artículo, la mayoría de los EVD deberían contener el sílabo, las presentaciones de las clases, las calificaciones y publicación de avisos, ya que son de uso común y se sugiere que habría que examinar cada curso para ver que otras herramientas serían útiles y que elementos hay que mejorar en beneficio del aprendizaje de nuestros alumnos. Esto último plantea las intenciones de alcanzar una mejor calidad.

Este es el primer estudio que trata sobre los usos de aulas virtuales en nuestra Universidad y en una
Facultad de Medicina peruana que puede servir de base para futuras investigaciones. Invito a las personas que enviaron la carta a llevarlas a cabo.

Carlos Orellano Médico y Cirujano.

Coordinador operativo de los Espacios Virtuales para la Docencia. Facultad de Medicina Alberto Hurtado. Universidad Peruana Cayetano Heredia.

\section{REFERENCIAS BIBLIOGRÁFICAS}

1. Orellano C. Uso de los espacios virtuales para la docencia en cursos de pregrado de Medicina. Rev Med Hered. 2012; 23(3):188-192.

2. Peña-Sarmiento $M$, Avendaño-Prieto BL. Evaluación de la implementación del aula virtual en una Institución de Educación Superior. Suma Psicológica. 2006; 13(2): 173-192.

Recibido: 28/01/13 